\title{
Ovarian Medulla
}

National Cancer Institute

\section{Source}

National Cancer Institute. Ovarian Medulla. NCI Thesaurus. Code C33245.

The central portion of the ovary, composed of loose connective tissue that contains blood vessels, lymph vessels and nerves. 